\title{
Sizes and size distributions of nanoparticles: Causes of differences in results obtained by transmission electron microscopy and small angle $X$-ray scattering
}

\author{
D. O. Shvedchenko, V. V. Volkov, E. I. Suvorova \\ A. V. Shubnikov Institute of Crystallography of Russian Academy of Sciences, \\ Leninsky pr., 59, 119333 Moscow, Russia \\ dmitrymephi@gmail.com, vvo@ns.crys.ras.ru, suvorova@ns.crys.ras.ru
}

PACS 61.05cf + 61.46.Df + 68.37.Lp

DOI 10.17586/2220-8054-2017-8-4-512-522

\begin{abstract}
Comparative size and structure characterization of silver and selenium nanoparticles obtained and stabilized in different polymer solutions was performed by transmission electron microscopy (TEM) and small-angle X-ray scattering (SAXS). Effects of instrumental properties, nature of the samples, data collecting and data processing on accuracy of measurements are highlighted and summarized. Numerical differences in the mode diameter values derived from the TEM and SAXS data were found to have different sources. The SAXS results can be misleading in case of small particles (2-4 nm), for instance, Ag nanoparticles formed and stabilized in some aqueous polymer solutions due to instrumental limits, while TEM can provide sufficient statistics on such nanoparticles. SAXS is efficient in characterization of size distributions for soft Se-polymer composite particles of 20 to $100 \mathrm{~nm}$ in diameter. TEM is mandatory for investigating the chemical and phase composition of particles in mixtures, and their formation mechanism.
\end{abstract}

Keywords: nanoparticles, selenium, silver, transmission electron microscopy, small-angle X-ray scattering, size distribution.

Received: 26 July 2017

Revised: 5 August 2017

\section{Introduction}

The chemical, physical, bio- and medical properties of different kinds of particle suspensions are strongly dependent on the particle sizes and the shape of their distributions. Transmission electron microscopy (TEM) and small-angle X-ray scattering (SAXS) remain the main techniques for characterizing nanoparticle sizes and their distributions. On this issue, we refer to reviews of Chu \& Liu [1] and Pedersen [2] with extensive analysis of principles and applications of different scattering techniques.

The main issue of experimental articles on the nanoparticle sizes and distributions is to show the best agreement between data in the wide range from a few $\mathrm{nm}$ to microns obtained by various scattering techniques and TEM. We argue that in general this approach is wrong since first, it does not consider the different ways that incident beams interact with matter. In this connection, it is worthwhile to mention an example of remarkably different size distributions and the mode diameter derived from the small-angle X-ray scattering and small-angle neutron scattering (SANS) curves obtained from the same sample as shown by Pedersen [2]. The explanation was that the neutrons "see" a high-density core while the X-rays "see" a low-density shell. Therefore, the size distribution histograms from SANS and SAXS for particles composed by copolymers with hydrophobic and hydrophilic chains or core-shell structures will describe different components of the same particles.

The fundamental difference of scattering methods like SAXS from TEM is the need of preliminary knowledge or assumption of shape, structure of particles in order to derive the sizes from the scattering curves. TEM is a direct method for imaging the particles and measuring their sizes over a wide range of diameters from $1 \mathrm{~nm}$ to a few tens nm with a capability to extract information of the chemical/phase composition of the particles and their crystalline character in polydisperse and multiphase systems. SAXS operates in terms of scattering intensities from objects depending on the scattering angles and thus is an indirect method of measuring sizes. Therefore, analysis of SAXS data contains a comparison with scattering model functions to fit experimental and simulated intensity curves.

In this paper, we show the advantages for a combination of the methods to obtain comprehensive information about particles in solutions, their distributions with high reliability and reveal the sources of inevitable errors in statistical analyses of particles in both mono- and polydisperse systems. Therefore, the goals of the paper are to: (1) highlight and summarize the main origin (instrumental, sampling, data collection and processing) of errors of these two methods, (2) show the ways to minimize the errors of measurements, (3) present the results of the TEM and SAXS investigation for two entirely different polydisperse systems of Ag and Se nanoparticles synthesized and stabilized in polymer solutions. We compare the volume fractions of the smallest particles (below $5 \mathrm{~nm}$ in 
diameter) measured by TEM and SAXS as well as volume fractions of particles with diameters at the large-size tails of distributions. For this purpose, we applied for the first time a new method of particle recognition and size measurement described in [3]. This automatic method based on approximation of the calculated distribution of grey level to the real distribution in (S)TEM images allowed us to increase the number of measured particles in comparison with the manual analysis.

\section{Theoretical background}

\subsection{SAXS}

The scattering intensity from the mixture of different particles may be represented as a sum of partial intensities from several non-interacting polydisperse components (polydisperse systems of particles having in the frame of the component the same shape but different sizes) weighted by their volume fractions [4,5]:

$$
I(s)=\sum_{k=1}^{K} V_{k} I_{k}(s)
$$

where $\mathrm{V}_{k}\left(\mathrm{~V}_{k}>0\right)$ and $\mathrm{I}_{k}(\mathrm{~s})$ are the volume fraction and scattering intensity from the k-th component, respectively, $\mathrm{K}$ is the number of components (usually, this number is chosen in the range of 2 to 4 ), each having its own analytical distribution with parameters to be determined. The characteristics of partial distributions were parameterized and restored along with their volume fractions using a non-linear least squares fitting procedure.

For the given particle shape and known normalized scattering intensity of a particle $i(s R)(s=0, i(0)=1)$, the scattering intensity $I_{k}(s)$ from the $k$-th component is determined as:

$$
I_{k}(s)=T_{k}(s) \cdot \int_{0}^{\infty} N_{k}(R) \cdot\left[v_{k}(R) \cdot \Delta \rho_{k}(R)\right]^{2} \cdot i_{k}(s, R) \cdot T_{k}(s) \cdot d R,
$$

where the scattering vector $s=(4 \pi / \lambda) \sin \theta,\left(\lambda\right.$ is the wavelength, and $2 \theta$ is the scattering angle), $N_{k}(R)$ is a function of size distributions, $\Delta \rho_{k}(R), v_{k}(R)$ and $i_{k}(s, R)$ are the contrast (the difference between the average electron density of the particle and average density of the environment), volume and normalized scattering intensity (the square of the form-factor) of the particle of the radius $R\left(i_{0 k}(0, R)=1\right), T_{k}(s)$ is a structure factor describing the interference between particles for the $k$-th component in the Percus-Yevick approximation [6]. We assume or learn from TEM that the components, i.e. the particles, are mainly spherical and have different sizes. The equation (1) can be written in the normalized form as:

$$
I_{k}(s)=V_{k} \frac{\int_{0}^{\infty} D_{k}(R) v_{k}(R)\left[\Delta \rho_{k}(R)\right]^{2} i_{0 k}(s, R) d R}{\int_{0}^{\infty} D_{k}(R) d R}
$$

if we take the total volume of the components as:

$$
V_{k}=\int_{0}^{\infty} v_{k}(R) N_{k}(R) d R
$$

The total volume distribution function $D_{k}(R)=N_{k}(R) v_{k}(R)$ can be normalized to expression

$$
\int_{0}^{\infty} D_{k}(R) d R=1
$$

The analytical expression of the $D_{k}(R)$ is a distribution with the average particle radius $R_{0 k}$ and the halfwidth $\Delta R_{k}$ :

$$
D_{k}(R)=G\left(R, R_{0 k}, \Delta R_{k}\right)=\left(\frac{z+1}{R_{0 k}}\right)^{z+1} \frac{R^{z}}{\Gamma(z+1)} \exp \left[-\frac{(z+1) R}{R_{0 k}}\right], \quad z=\left(\frac{R_{0 k}}{\Delta R_{k}}\right)^{2}-1 .
$$

The form factor in this work is the scattering amplitude from spherical particles of radius $R$ because electron microscopy has shown that the particles are spherical. 
To determine the volume fractions and other parameters characterizing the mixture, the experimental scattering intensity $I_{\text {exp }}(s)$ should be decomposed into the partial functions (3). This can be done by a non-linear minimization based on the squared residual target function:

$$
\chi^{2}=\sum_{j=1}^{N}\left\{\left[c I\left(s_{j}\right)-I_{\exp }\left(s_{j}\right)\right] / \sigma\left(s_{j}\right)\right\}^{2}
$$

where $N$ is the number of experimental points and $\sigma(\mathrm{s})$ denotes the statistical error. The use of the scale factor:

$$
c=\frac{\sum_{j=1}^{N} I\left(s_{j}\right) I_{\exp }\left(s_{j}\right) / \sigma^{2}\left(s_{j}\right)}{\sum_{j=1}^{N}\left(I\left(s_{j}\right) / \sigma\left(s_{j}\right)\right)^{2}}
$$

allows one to fit the experimental data in a relative scale.

In order to fit experimental $I_{\exp }$ and simulated $I_{\bmod }$ intensities, minimization of the non-linear quadratic functional with the $V_{k}, R_{0 k}, \Delta R_{k}$, and $T_{k}(s)$ parameters should be performed:

$$
\begin{aligned}
& \min _{V_{k}, R_{0, k}, \Delta R_{k}, \Delta \rho_{k}}\left\{\frac{\sum_{i=1}^{N}\left[\left(I_{\exp }\left(s_{i}\right)-c \cdot I_{\bmod }\left(s_{i}\right)\right) \cdot W\left(s_{i}\right)\right]^{2}}{\sum_{i=1}^{N}\left[I_{\exp }^{2}\left(s_{i}\right) \cdot W^{2}\left(s_{i}\right)\right]}\right\}, \quad k=1, \ldots, K, \\
& W\left(s_{i}\right)=\left[\tilde{I}_{\exp }\left(s_{i}\right)\right]^{p} / \tilde{I}_{\exp }\left(s_{i}\right), \quad p=1,-\frac{1}{2},-\frac{2}{3},-\frac{3}{4},
\end{aligned}
$$

where $N$ is a number of experimental points, $W(s)$ is a weighting function calculated from smoothed experimental data and used to provide the optimum intensity range for all experimental $s$ values. In this work, $p=1 / 2$ was used as an optimal value obtained from the simulation, c is a fitting factor for $I_{\text {exp }}(s)$ and $I_{\bmod }(s)$ (7) calculated before the minimization of non-linear quadratic functional. The minimization was performed using the multivariant optimization program package OPTIS as it was applied in the program MIXTURE [4].

\subsection{TEM}

Imaging in TEM is provided through the mass-thickness contrast produced by incoherent (Rutherford) elastic scattering of electrons for both crystalline and non-crystalline materials. The cross-section for elastic scattering is a function of the atomic number $(Z)$, therefore the samples containing elements with higher $Z$ will scatter more electrons than the low- $Z$ samples, besides the electron scattering increases from thicker areas. Therefore, the mass-thickness contrast is a universal mechanism of imaging for crystalline and non-crystalline objects.

The definition of the contrast $K$ is the difference in intensity $\Delta I$ between two adjacent areas [7]:

$$
K=\frac{I_{1}-I_{2}}{I_{2}}=\frac{\Delta I}{I_{2}}=1-e^{-Q \Delta t} \cong Q \Delta t=N_{0} \sigma \rho \Delta t,
$$

where $Q$ is the total cross section for scattering from the sample, $N_{0}$ is Avogadro's number, $\rho$ is the density, $\Delta t$ is a change in thickness, and $\sigma$ is the single-atom scattering cross section dependent on energy of incident electrons $E_{0}(k e V)$, a scattering angle $\theta$, and proportional to atomic number square $Z^{2}$ (if the effects of electron cloud are ignored).

The number of transmitted electrons, $n$, through the sample with mass-thickness $\rho t$ can be approximated by the expression:

$$
n=n_{0} e^{-N \sigma \rho t}
$$

The latter formula means the decrease in the number of transmitted electrons with sample thickness, that is, the increase in the number of scattered electrons by thick samples.

High angle annular dark-field (HAADF) scanning TEM (STEM) provides a way of imaging mainly $Z$-contrast by collecting incoherently scattered electrons and thus possesses a strong compositional sensitivity. Diffraction contrast is a feature of crystalline materials to investigate defects, and phase contrast applied in high resolution TEM is well suited to the study of atomic structure of materials. All these types of contrast in (S)TEM are used for particle analysis and provide comprehensive information of their morphology and structure. 


\subsection{Origin of measurement differences and errors in SAXS and TEM}

The fundamental difference between TEM and SAXS is in the nature of beams and their interaction with matter. The electrostatic Coulomb potential is the scattering matter for electron beams while the electric charge density is the scattering matter for X-rays. This difference leads to the different ways of forming intensity distributions when electron beams and X-rays penetrate through the same sample. There is a direct mathematical link between the $\mathrm{X}$-ray and electron scattering through scattering amplitudes.

Using Thomson scattering equations of electromagnetic waves on the electric charge we can obtain the dependence of the amplitude of X-ray scattering $f_{X}(\vec{s})$ from the electron density $\rho_{e}(\vec{r})$ and scattering vector $\vec{r}$ :

$$
f_{X}(\vec{s})=r_{0} \iiint \frac{\rho_{e}(\vec{r})}{e} e^{i(\vec{s} \vec{r})} d^{3} \vec{r}
$$

where $r_{0}=\frac{1}{4 \pi \varepsilon_{0}} \cdot \frac{e^{2}}{m_{0} c^{2}}$ is electron radius.

Amplitude scattering of electrons in Born approximation can be written as:

$$
f_{e}(\vec{s})=\left(\frac{2 \pi m e}{h^{2}}\right) \iiint \varphi_{a} t(\vec{r}) e^{i(\vec{s} \vec{r})} d^{3} \vec{r} .
$$

Using Poisson equation $\Delta \varphi_{a t}=\frac{\rho_{a t}}{\varepsilon_{0}}$, the link between $f_{p}$ and $f_{e}$ can be derived. If the atomic charge density $\rho_{a t}=\rho_{n}-\rho_{e}$, then the nuclear charge density is:

$$
\rho_{n}(\vec{r})=Z e \delta(\vec{r})=\frac{1}{(2 \pi)^{3}} \iiint e Z e^{-i(\vec{s} \vec{r})} d^{3} \vec{s} .
$$

Fourier transform is used to derive the electron density and atom potential through X-ray scattering and electron amplitudes:

$$
\begin{gathered}
\rho_{e}(\vec{r})=\frac{1}{(2 \pi)^{3}} \iiint \frac{e}{r_{0}} f_{X}(\vec{s}) e^{-i(\vec{s} \vec{r})} d^{3} \vec{s}, \\
\varphi_{a t}(\vec{r})=\frac{1}{(2 \pi)^{3}} \iiint \frac{h^{2}}{2 \pi m e} f_{e}(\vec{s}) e^{-i(\vec{s} \vec{r})} d^{3} \vec{s} .
\end{gathered}
$$

Using the Poisson equation, we obtain:

$$
-s^{2} \frac{h^{2}}{2 \pi m e} f_{e}(\vec{s})=-\frac{1}{\varepsilon_{0}}\left(e Z-\frac{e}{r_{0}} f_{X}(\vec{s})\right) .
$$

Finally, taking into account that $s=4 \pi \frac{\sin \theta}{\lambda}$, the link between the electron and X-ray scattering amplitudes is written as:

$$
f_{e}=\frac{1}{4 \pi \varepsilon_{0}} \cdot \frac{m e^{2}}{2 h^{2}} \cdot \frac{Z-f / r_{0}}{\left(\frac{\sin \theta}{\lambda}\right)^{2}} .
$$

This formula shows the linear dependence between the electron and X-ray scattering amplitudes and their ratio is $f_{e}: f_{X}=10^{3}: 1$. Direct proportionality between $f_{e}$ and $Z$ leads to $\mathrm{Ag}$ nanoparticles with high $Z$ on/in the carbon (low $Z$ ) substrate having a significant contrast in TEM images. While SAXS is able to provide reliable information about small particles of 1 to $1.5 \mathrm{~nm}$ in diameter if their concentrations are high enough and this scattering intensity exceeds that by some density fluctuations of solvents and organic material agglomerations.

Difficulties in recognition of the smallest particles from SAXS data in the range of the sizes from 1 to $5 \mathrm{~nm}$ in the heterogeneous polymer solutions is strongly dependent upon the distribution profile on the shape of the scattering curve at angles greater than $\mathrm{s}=0.5-1 \mathrm{~nm}^{-1}$, which is largely dependent on the scattering from other small inhomogeneities in the solution. This may lead to false peak appearance in the size distribution or to its shift to larger or smaller sizes.

In Table 1, we highlight and summarize the causes of errors due to instrumental properties, features of the samples, data collecting and data processing. The main conclusion from Table 1 is that TEM is most efficient for local analysis since it performs imaging and composition/phase identification, while SAXS produces integral information which depends on the initial hypotheses as to the shape, sizes and state of matter.

There is no methodological limitation to reveal polydisperse distributions of the nm-sized particles using modern TEM equipment, and the reliable statistics can be reached by counting the sufficient number of particles using the relevant software, which significantly reduces the time-consuming efforts.

SAXS can be very useful (fast and reliable) for monitoring the monodispersed particles distributions or a narrow polydispersity in industrial laboratories with the possibility of measuring the total surface of samples. 
TABLE 1. Summary of origin of errors in sizing particles due to instrumental properties, sample features, and data collecting and processing

\begin{tabular}{|l|l|l|}
\hline & TEM & SAXS \\
\hline Instrumental & $\begin{array}{l}\text { Images as particle projections. } \\
\text { Small volume of material under investiga- } \\
\text { tion (sample thickness } \leq 500 \mathrm{~nm} \text { ) } \\
\text { Loss of contrast when the fine beam is used. }\end{array}$ & $\begin{array}{l}\text { Divergence of the X-ray beam and the } \\
\text { spread of scattering angles. } \\
\text { Halo of parasitic radiation around the direct } \\
\text { beam. } \\
\text { Loss of intensity when the fine beam is } \\
\text { used. } \\
\text { Parasitic scattering (the need of monochro- } \\
\text { mators) }\end{array}$ \\
\hline Sample & $\begin{array}{l}\text { Deterioration of particle contrast due to } \\
\text { burying in the polymer film. } \\
\text { Deterioration of particle contrast due to car- } \\
\text { bon contamination because of electron irra- } \\
\text { diation of organic material. } \\
\text { Possible deformation of the particle shape } \\
\text { due to drying }\end{array}$ & $\begin{array}{l}\text { Widely different particle shapes provide } \\
\text { only slightly different scattering curves. } \\
\text { Particles with different composition give } \\
\text { similar scattering curves. } \\
\text { Scattering by large particles (above 300 nm) } \\
\text { Double Bragg reflection form crystallites. }\end{array}$ \\
\hline $\begin{array}{l}\text { Data collecting } \\
\text { processing }\end{array}$ and & $\begin{array}{l}\text { Sampling is not random and is often deter- } \\
\text { mined by the nice appearance of particles. } \\
\text { Great operating skill is required to get prop- } \\
\text { erly focused images of nanoparticles espe- } \\
\text { cially in STEM images. } \\
\text { Time consuming manual measurements or } \\
\text { inappropriate software leading to insuffi- } \\
\text { cient statistics. }\end{array}$ & $\begin{array}{l}\text { Assumptions about the form of the } \\
\text { distribution functions are required. } \\
\text { All generalized definitions are derived as- } \\
\text { suming that all particles in the sample are } \\
\text { identical. } \\
\text { Similar scattering curves for different diam- } \\
\text { eter distribution functions. }\end{array}$ \\
\hline
\end{tabular}

\section{Experimental: Materials and Methods}

Silver nanoparticles were synthesized by the chemical reduction of $0.3 \mathrm{M}$ silver nitrate in aqueous solutions of 2 wt. \% 2-deoxy-2-methacrylamido-D-glucose (MAG)/2-(dimethylamino) ethyl methacrylate (DMAEMA) copolymers or pure DMAEMA and MAG homopolymers at room temperature and pressure. All synthetic details are given elsewhere $[8,9]$. Selenium particles were obtained by reduction of selenious acid using ascorbic acid and stabilized by poly(2-acrylamido-2-methylpropane sulfonic acid) (PAMPS) or by polyvynilpyrrolidon (PVP) in aqueous solutions. Details of the process are described elsewhere [10,11].

TEM-HRTEM-STEM and X-ray energy dispersive spectrometry (chemical miscoanalysis) investigation of Ag nanoparticle samples - polymer water and Se nanoparticle - polymer water suspensions (1.0 - 2.0- $\mu$ 1 droplets) lying on carbon films $/ \mathrm{Cu}$ grids after drying were investigated by transmission (TEM), scanning transmission electron microscopy (STEM) and X-ray energy dispersive spectroscopy in a FEI Tecnai Osiris microscope (200 kV X-FEG field emission gun). The particle diameters were measured using the new method [3] based on approximation of the calculated distribution of grey level to the real distribution in (S)TEM images providing the best accuracy in measurements of the particle diameters in contrast to algorithms based on image thresholding approach.

Small-angle X-ray scattering (SAXS) measurements were carried out on a laboratory diffractometer "AMUR$\mathrm{K}$ " in the Institute of Crystallography, Moscow [12] at the $\mathrm{Cu}-\mathrm{K}_{\alpha}$ wavelength $\lambda=0.154 \mathrm{~nm}$ using the Kratky-type collimation system. The linear position-sensitive proportional detector used provided the range of the scattering vector modulus $0.11<s<15 \mathrm{~nm}^{-1}(s=4 \pi \sin \theta / \lambda$, where $2 \theta$ is the scattering angle). Solution samples were placed in a $1 \mathrm{~mm}$ quartz capillary with $0.01 \mathrm{~mm}$ walls. The obtained scattering profiles were preprocessed by the program package PRIMUS [13] to correct the water scattering and instrumental effects [14]. The volume particle distributions were obtained with the MIXTURE program $[4,13]$ approximating the particles as spheres. The model volume distributions were presented as three superimposed Schulz distributions with different parameters chosen by progressive iterations during fitting the experimental and calculated scattering intensities. 


\section{Results and Discussion}

\subsection{Ag particles}

(S)TEM images of silver particles obtained in MAG-DMAEMA copolymer solutions with DMAEMA mole fractions 12, 44, and $100 \%$ are shown in Fig. 1(a,b,c) with the experimental scattering curves and data processing.
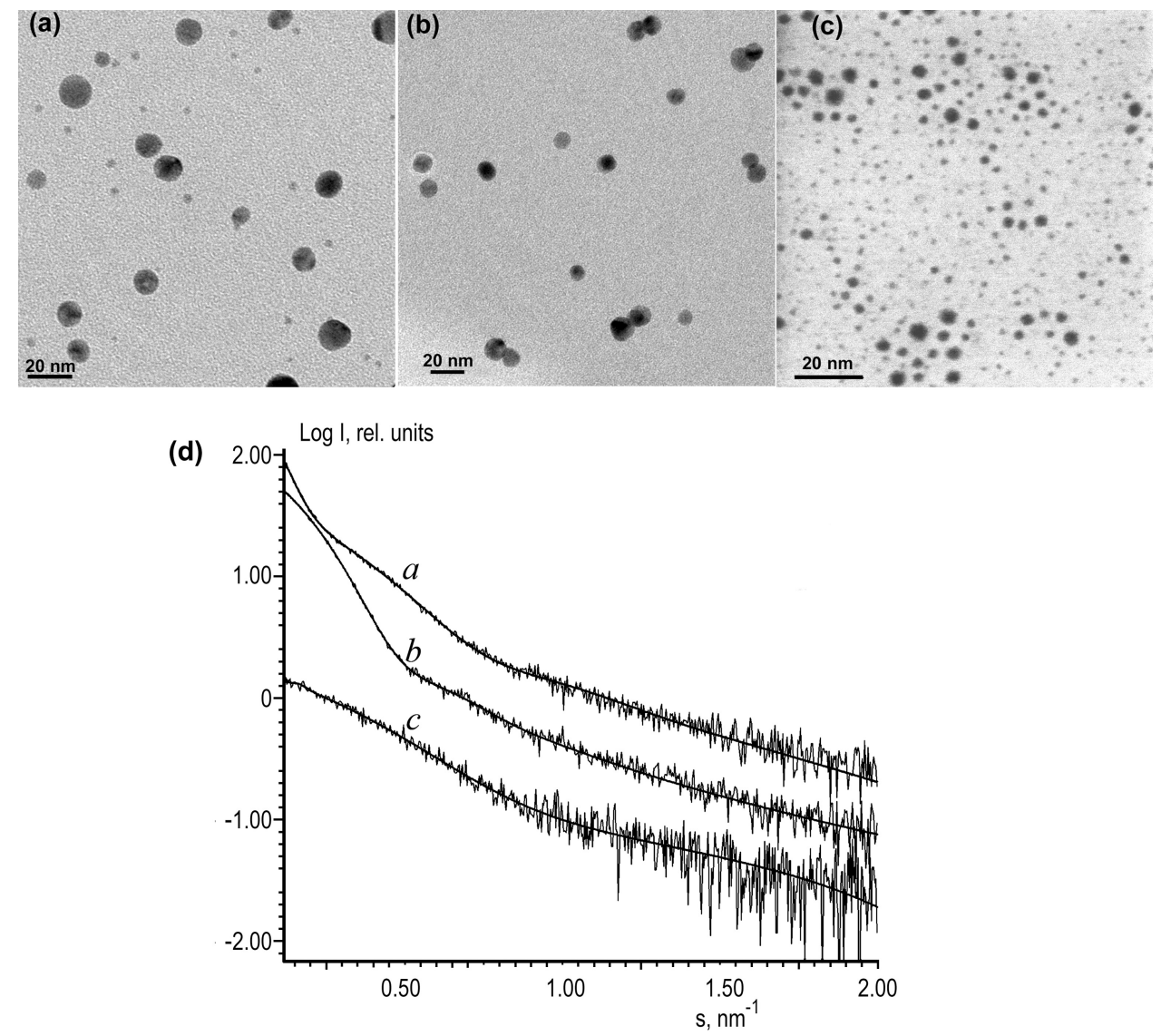

FIG. 1. TEM images (a, b, c) of Ag particles, experimental and approximated (solid lines) SAXS curves (d) obtained from the samples of Ag particles in polymer solutions: MAG-12\% DMAEMA (a), MAG-44 \% DMAEMA (b) and pure DMAEMA (c)

The SAXS volume distribution functions and the volume and particle distributions obtained by TEM for Ag particles in three samples with different copolymers are superimposed in Fig. 2.

The mode diameter values of Ag particles measured by SAXS and TEM/STEM with standard deviations are given in Table 2. The absolute difference in these values is $4 \mathrm{~nm}$ and the relative can reach $60 \%$. We can see the shift of the peaks in TEM volume histograms relative to SAXS maximums in volume distribution functions to the side of smaller sizes for the samples with the single mode distributions and to the side of larger diameters for the double mode distributions. The largest difference close to $60 \%$ is observed for the particles in pure DMAEMA homopolymer. We reported earlier [9] that the particles in this sample had the smallest diameters with the mode diameter between 2 and $4 \mathrm{~nm}$, which is the principal limit for SAXS measurements. The difference in mode diameter for two copolymers does not exceed $29 \%$. The origin of higher value of mode diameter revealed by SAXS for particles in pure DMAEMA and MAG-44 \% DMAEMA copolymer can be associated with the formation of a protective polymer shell around particles, which also contributes to small-angle scattering. It is quite probable that a protective shell was formed for particles in MAG-12\% DMAEMA solution, however, the effect of size averaging in polydisperse system with bimodal distribution resulted in some shift of the SAXS volume distribution function peak towards smaller diameters. 

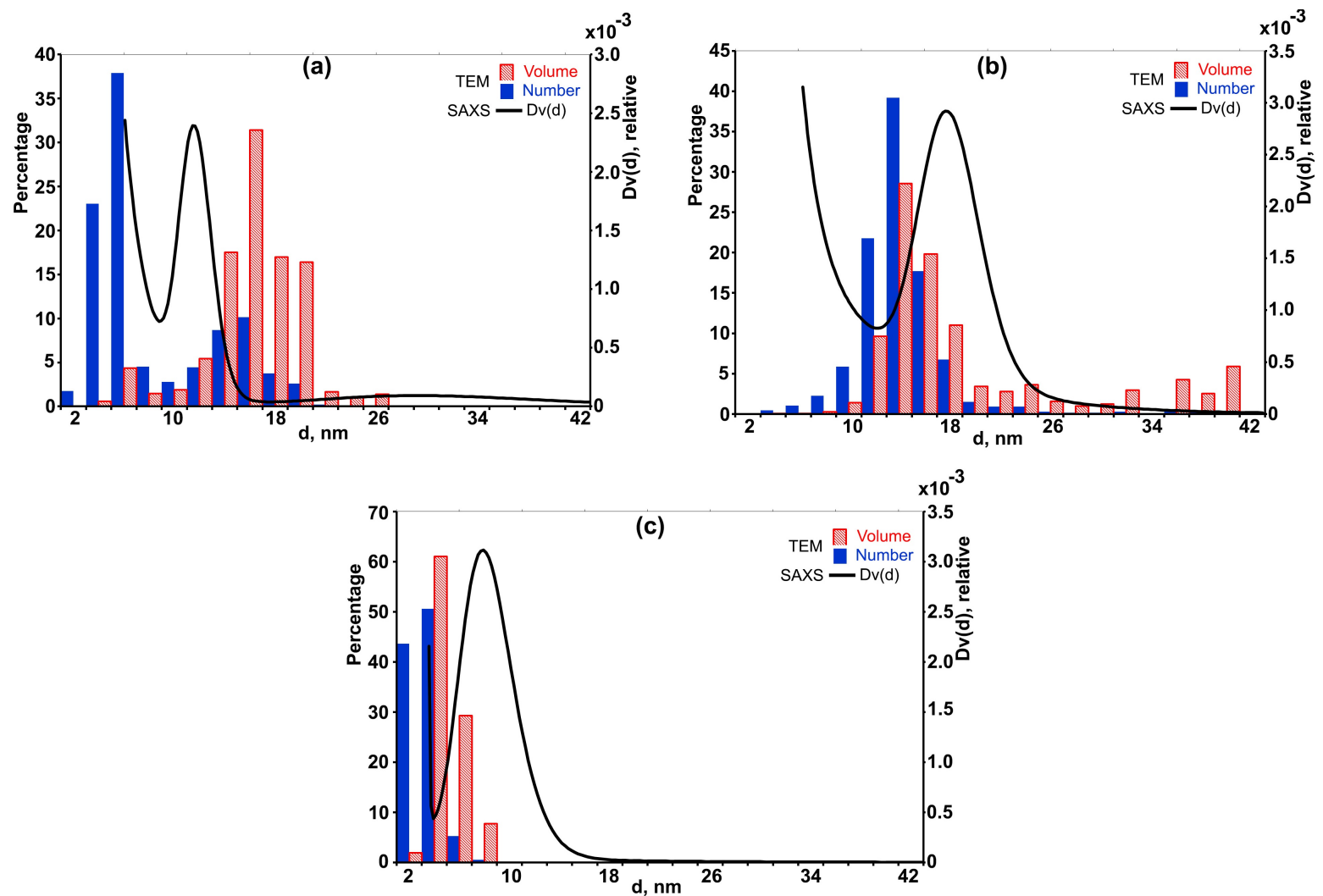

FIG. 2. Volume distribution functions (solid lines) superimposed with histograms of volume and particles distributions vs. particle diameters in two copolymer solutions MAG-12\% DMAEMA (a), MAG-44 \% DMAEMA (b), and pure DMAEMA (c)

TABLE 2. Mode diameters of Ag particles in volume distributions obtained by SAXS and TEM/STEM

\begin{tabular}{|c|c|c|}
\hline \multirow{2}{*}{ Sample } & \multicolumn{2}{|c|}{ Mode diameter, $\mathrm{nm}$} \\
\cline { 2 - 3 } & TEM & SAXS \\
\hline $\mathrm{Ag}$ in MAG+12 \% DMAEMA & $17 \pm 2$ & $12 \pm 2$ \\
\hline $\mathrm{Ag}$ in MAG+44.5 \% DMAEMA & $13 \pm 2$ & $17 \pm 2$ \\
\hline $\mathrm{Ag}$ in pure DMAEMA & $3 \pm 2$ & $7 \pm 2$ \\
\hline
\end{tabular}

\subsection{Se particles}

TEM images of selenium particles obtained in aqueous PAMS and PVP polymer solutions are shown in Fig. 3(a,b) with their experimental and approximated (solid lines) SAXS intensity curves (Fig. 3(c)). The SAXS volume distribution functions and the volume and particle distributions obtained by TEM for Se particles in PAMS and PVP polymers are superimposed in Fig. 4. The accuracy of distribution parameters for the 3-component models was $2-8 \%$ (in diameter) and $20-40 \%$ for the distribution half-width. The mode diameters of particles measured with TEM and SAXS with standard deviations are listed in Table 3.

TABLE 3. Mode diameters of particles in PVP and PAMS solutions obtained from SAXS scattering

\begin{tabular}{|c|c|c|}
\hline \multirow{2}{*}{ Sample } & \multicolumn{2}{|c|}{ Mode diameter, nm } \\
\cline { 2 - 3 } & TEM & SAXS \\
\hline Se+PVP & $53 \pm 2$ & $20 \pm 3$ \\
\hline Se+PAMS & $65 \pm 2$ & $29 \pm 4$ \\
\hline
\end{tabular}



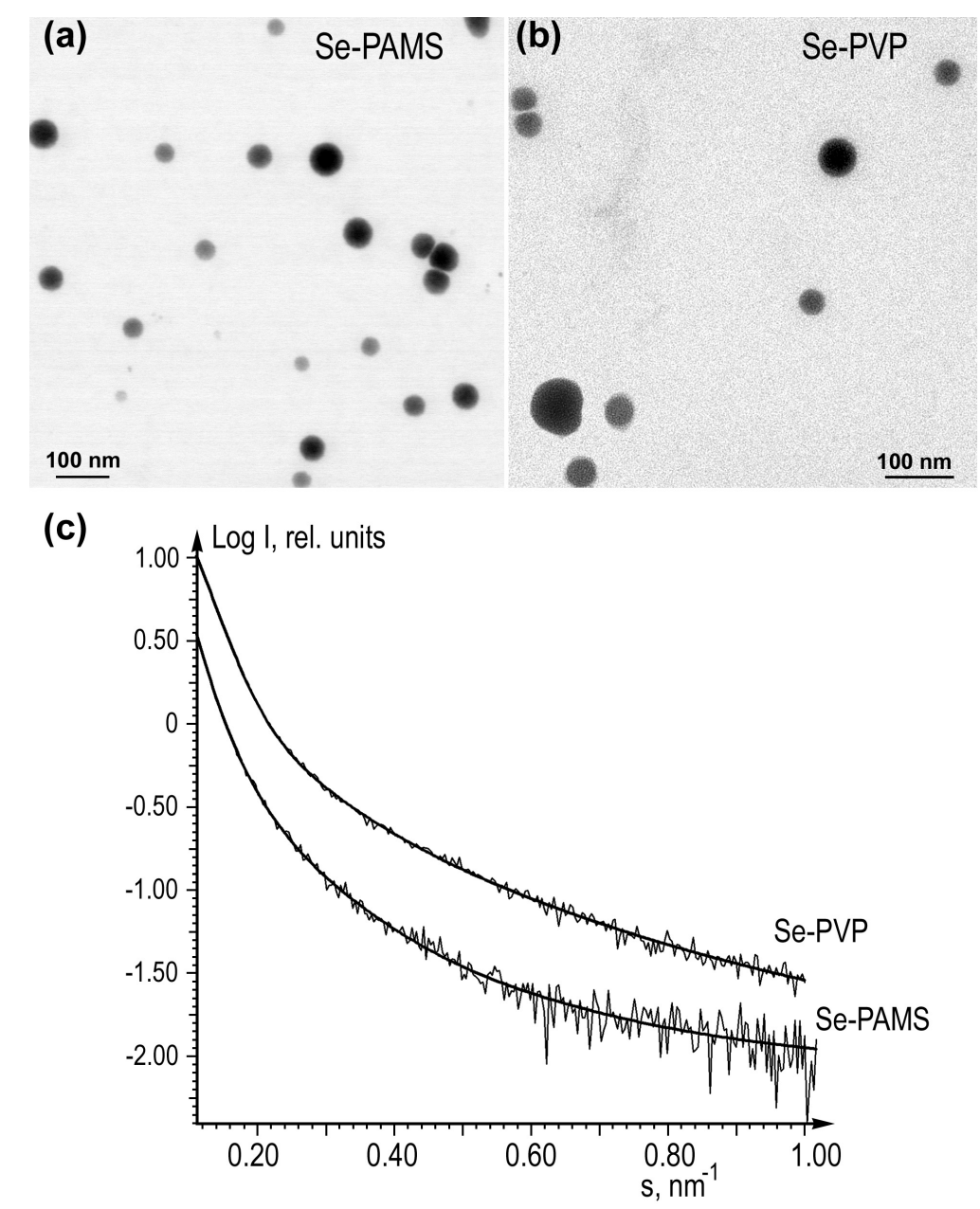

FIG. 3. TEM images of Se particles formed in PAMS (a) and PVP (b) solutions, the experimental and approximated (solid lines) SAXS intensity curves (c)


FIG. 4. Volume distribution functions (solid curves) superimposed with histograms of volume and particles distributions vs. particle diameters in Se + PAMS (a), and Se + PVP (b) samples 
In contrast to Ag particles, the mode diameters for Se particles differ by more than two-fold in SAXS and TEM measurements. The explanation of this result is in nature of particles. TEM and STEM images (Fig. 5(a,b)) show groups of 2 or 3 deformed particles. The deformation occurred due to surface tension when the TEM sample of water suspension was drying on a carbon film. Obviously, the deformation of particles occurred in the lateral direction when the particles met each other and in the vertical direction under surface tension and gravity, and Se particles became oblate spheroids that are spheroids flattened at the poles. Thus, in the case of soft Se particles, TEM provides only projections of spheroids with larger diameters than those of spherical particles, while SAXS determines the true mode diameters of Se particles.



FIG. 5. TEM image of Se particles from the PAMS solution with strong and weak contrast of particles (a), three crystalline Se particles with strong contrast (b), and the corresponding SAED pattern taken from one of the particles (indicated by arrow) along the $\left[\begin{array}{lll}-1 & 8 & 12\end{array}\right]$ direction in monoclinic Se

Cryogenic TEM can be used to investigate soft Se particles, which can be frozen in a thin water layer, or to image particles located in thin edges of vacuum-dried polymer films. In both cases, the statistics are quite low due to small number of available particles and radiation damage which has a significant effect.

But first, not all grouped Se particles are deformed and second, Se particles in the same sample have different contrast (Fig. 5(a)). Only TEM answers the question as to why Se particles have different structure. They can be crystalline with monoclinic structure, which was discovered by electron diffraction (Fig. 5(b,c)) and these particles are dark in TEM images. They keep the spherical shape and do not deform under surface tension. While other particles do not have crystalline structure, and are not always pure Se particles but Se/polymer composite particles with a different content of polymer inside particles (Fig. 6). X-ray energy dispersive spectrometry (chemical microanalysis) showed different amount of polymer material inside of particle with Se.

Earlier [15], we proposed the mechanism of formation for Se/polymer composite particles and that their rigidity and density differ from the crystalline Se and amorphous Se particles. Also, it was concluded that such differences can influence the biological and medical properties of the particles.

\section{Conclusion}

In summary, silver and selenium particles obtained and stabilized in different polymer solutions were studied by SAXS and TEM to characterize the size distributions and structure. It was found that numerical difference in the mode diameter values derived from the TEM and SAXS measurements can be caused by various reasons. The SAXS results can be misleading in the case of small particles with the mode diameter of about $2-4 \mathrm{~nm}(\mathrm{Ag}$ particles in the pure DMAEMA polymer solution) due to instrumental limits and TEM can give unambiguous information on the size distribution with sufficient statistics.

Averaging the sizes in colloid systems with bimodal size distribution by SAXS shifts the maximum into some intermediate position as for Ag particles in MAG-12 \% DMAEMA suspension.

Different mode diameter values for nanoparticles coated by the polymer shells measured by X-rays scattering and TEM images can be explained by low contrast of thin polymer shell in TEM images.

SAXS is efficient in characterization of soft particles like Se/polymer composite particles in the range of diameters from 20 to $100 \mathrm{~nm}$. TEM characterization of such soft particle leads to artificial increase of diameters 




FIG. 6. STEM image and Se/C chemical map with the ratio profile across the Se/PAMS composite particles

due to particle deformation and shape transformation from spherical to oblate spheroids. At the same time TEM study is mandatory to reveal the chemical and structural features of such particles.

\section{Acknowledgments}

The authors are thankful to Prof. P. A. Buffat (École Polytecnique Fédéral de Lausanne) for the help in electron microscopy analysis, discussions and comments to the paper.

\section{References}

[1] Chu B., Liu T. Characterization of nanoparticles by scattering techniques. J. Nanopart. Res., 2000, 2, P. $29-41$.

[2] Pedersen J.S. Analysis of small-angle scattering data from colloids and polymer solutions: modeling and least-squares fitting. Adv. Colloid. Interfac., 1997, 70, P. 171-210.

[3] Shvedchenko D.O., Suvorova E.I. A novel method of automated statistical analysis of polymer stabilized metal nanoparticles in electron microscopy images. Crystallogr. Rep.+, 2017, 62(5), P. 802-808.

[4] Svergun D.I., Konarev P.V., Volkov V.V., Koch M.H.J., Sager W.F.C., Smeets J., Blokhuis E.M. A small angle x-ray scattering study of the droplet-cylinder transition in oil-rich sodium bis(2-ethylhexyl) sulfosuccinatemicroemulsions. J. Chem. Phys., 2000, 113, P. 1651-1665.

[5] Svergun D.I. Small-angle scattering studies of macromolecular solutions. J. Appl.Cryst., 2007, 40, P. s10-s17.

[6] Percus J.K., Yevick G.J. Analysis of Classical Statistical Mechanics by Means of Collective Coordinates. Phys. Rev., 1958,110 , P. 1-13.

[7] Williams D.B., Carter C.B. Transmission Electron Microscopy. Plenum Press, New York, 1996, 729 pp.

[8] Nekrasova T.N., Zolotova Y.I., Nazarova O.V., Levit M.L., Suvorova E.I., Sirotkin A.K., Baklagina Y.G., Didenko E.V., Pautov V.D.,Panarin E.F. Silver nanocomposites based on (Co)polymers of 2-deoxy-2-methacrylamido-D-glucose, N-vinylamides, and aminoacrylates. Dokl. Chem., 2013, 446, P. 212-214.

[9] Shvedchenko D.O., Nekrasova T.N., Nazarova O.V., Buffat P.A., Suvorova E.I. Mechanism of formation of silver nanoparticles in MAG-DMAEMA copolymer aqueous solutions. J. Nanopart. Res., 2015, 17, P. 275.

[10] Valueva S.V., Kopeikin V.V., Kipper A.I., Filippov A.P., Shishkina G.V., Khlebosolova E.N., Rumyantseva N.V., Nazarkina Y.I., Borovikova L.N. Formation of Zero-Valence Selenium Nanoparticles in Polyampholyte Aqueous Solutions in the Presence of Redox Systems. Polym. Sci. Ser. B+, 2005, 47, P. 143-146. 
[11] Valueva S.V., Kipper A.I., Kopeikin V.V., Borovikova L.N., Lavrent'ev V.K., Ivanov D.A., Filippov A.P. Formation and Morphological Characteristics of Selenium-Containing Nanostructures Based on Rigid-Chain Cellulose Derivatives. Polym. Sci. Ser. A+, 2006, 48, P. 803-810.

[12] Mogilevsky L.Yu., Dembo A.T., Svergun D.I., Feigin L.A. Small-angle scattering diffractometer with single coordinates detector. Crystallogr. Rep.+, 1984, 29, P. 587-591.

[13] Konarev P.V., Volkov V.V., Sokolova A.V., Koch M.H.J., Svergun, D.I. PRIMUS: a Windows PC-based system for small-angle scattering data analysis. J. App.Cryst., 2003, 36, P. 1277-1282.

[14] Feigin L.A., Svergun D.I. Structure Analysis by Small-Angle X-Ray and Neutron Scattering. Plenum Press, New York, 1987,335 pp.

[15] Suvorova E.I., Klechkovskaya V.V. Effect of polymer matrix on structure of Se particles formed in aqueous solutions during redox process. Crystallogr. Rep.+, 2010, 55, P. 1116-1121. 